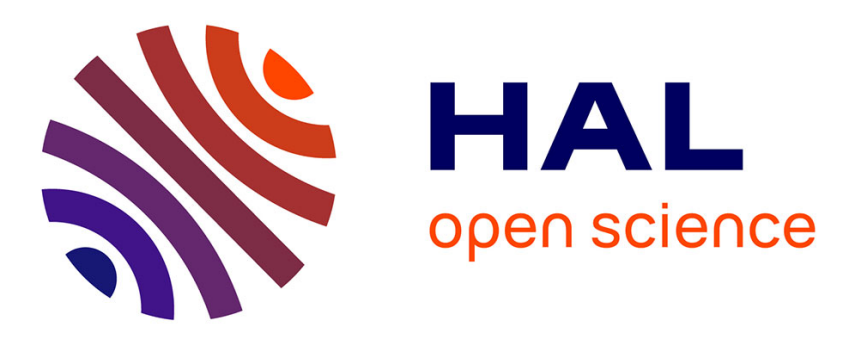

\title{
Regularity and uniqueness results for a phase change problem in binary alloys
}

Jean-Francois Scheid, Giulio Schimperna

\section{To cite this version:}

Jean-Francois Scheid, Giulio Schimperna. Regularity and uniqueness results for a phase change problem in binary alloys. 4th European Conference Elliptic and Parabolic Problems, Sep 2001, Gaeta, Italy. pp.475-484, 10.1142/9789812777201_0045 . hal-03116903

\section{HAL Id: hal-03116903 https://hal.science/hal-03116903}

Submitted on 20 Jan 2021

HAL is a multi-disciplinary open access archive for the deposit and dissemination of scientific research documents, whether they are published or not. The documents may come from teaching and research institutions in France or abroad, or from public or private research centers.
L'archive ouverte pluridisciplinaire HAL, est destinée au dépôt et à la diffusion de documents scientifiques de niveau recherche, publiés ou non, émanant des établissements d'enseignement et de recherche français ou étrangers, des laboratoires publics ou privés. 


\title{
Regularity and Uniqueness Results for a Phase Change Problem in Binary Alloys
}

\author{
Jean-François Scheid \\ Institut de Mathématiques Élie Cartan, Université de Nancy 1, \\ F-54506 Vandoeuvre-lès-Nancy Cedex, France \\ E-mail: scheid@iecn.u-nancy.fr \\ Giulio Schimperna \\ Dipartimento di Matematica, Università di Pavia \\ Via Ferrata, 1, I-27100 Pavia, Italy \\ E-mail: giulio@dimat.unipv.it
}

November 14, 2001

\begin{abstract}
An isothermal model describing the separation of the components of a binary metallic alloy is considered. A process of phase transition is also assumed to occur in the solder; hence, the state of the material is described by two order parameters, i.e., the concentration $c$ of the first component and the phase field $\varphi$. Existence of a solution to the related initial and boundary value problem has been proved in a former paper, where, anyway, uniqueness was obtained only in a very special case. Here some further regularity and uniqueness results are shown in a more general setting by use of an a priori estimates - compactness argument. A key point of the proofs is the analysis of the fine continuity properties of the inverse map of the solution-dependent elliptic operator characterizing one of the equations of the system.
\end{abstract}

Key words: phase separation, fourth order parabolic system, solution-dependent elliptic operator, constraint, variational formulation.

AMS (MOS) subject classification: 35K35, 35R35, 47H05, 74N25.

\section{Introduction and mathematical preliminaries}

In this paper, we aim to present some regularity and uniqueness results for the system

$$
\begin{aligned}
& \partial_{t} \varphi-\Delta \varphi=F_{1}(\varphi)+c F_{2}(\varphi), \\
& \partial_{t} c-\operatorname{div}(\mu(\varphi, c) \nabla w)=0, \\
& w \in-\Delta c+\beta(c)+\gamma(c)+g(\varphi),
\end{aligned}
$$


describing the diffusive separation of components in a binary metallic alloy possibly undergoing a phase transition phenomenon. In the above relations, the unknown $c$ represents the relative concentration of either of the components, while $\varphi$ is the phase parameter, with $\varphi=0$ denoting the solid, $\varphi=1$ the liquid, and $0<\varphi<1$ a mixture. Moreover, $F_{1}, F_{2}, \gamma$, and $g$ are smooth coupling terms whose meaning is outlined in the paper [4]. Finally, $\mu$ is the mobility coefficient, possibly depending on both the unknowns, but assumed to be nondegenerate, and $\beta$ is a maximal monotone graph guaranteeing the "physical" constraint $0 \leq c \leq 1$.

Let us point out that the system above has to be complemented with the Cauchy conditions for $\varphi$ and $c$ and with homogeneous Neumann boundary conditions for $\varphi$, $w$, and $c$. Under such assumptions, eqns. (1.1-1.3) have been studied in the paper [4], where existence and regularity results were proved for a variational formulation of that system. However, the question of uniqueness was solved just in the very particular case when $\mu$ is a constant. Hence, in this paper we deal with two more general settings, where the mobility $\mu$ may depend either on the sole unknown $\varphi$ or on both the unknowns $(\varphi, c)$, and we are able to show two distinct uniqueness and continuous dependence results. Indeed, both situations seem to be significant on the physical viewpoint [4, Introduction]. In order that the second result can be applied, anyway, we have to assume very strong smoothness properties of the solutions. In this concern, we actually prove a new regularity theorem for (1.1-1.3) that fits with the uniqueness setting at least for suitably small final times. The main analytical instrument on which we rely consists in a fine analysis of the continuity properties of the solution-dependent elliptic operators resulting from the nonconstant mobility $\mu$. This follows the lines of the work [1], where similar arguments were used for the study of the Cahn-Hilliard system with nonconstant mobility.

Let us now start by briefly presenting some mathematical notations and tools that will be used throughout the rest of the paper. Let $\Omega$ be a smooth, bounded, and connected domain in $\mathbb{R}^{3}$ (the situation in space dimension 2 is analogous and in dimension 1 is easier) and let $T>0$ be a given final time. Set $\Gamma:=\partial \Omega, \Sigma:=\Gamma \times(0, T)$, $Q_{t}:=\Omega \times(0, t)$ for $t \in(0, T]$, and $Q:=Q_{T}$. Set also $H:=L^{2}(\Omega)$ and $V:=H^{1}(\Omega)$ and endow the latter space with its usual scalar product. Identify $H$ and its dual, in order that the compact inclusion $H \subset V^{\prime}$ holds and $\left(V, H, V^{\prime}\right)$ form a Hilbert triplet. Finally, denote by $\langle\cdot, \cdot\rangle$ the duality pairing between $V^{\prime}$ and $V$, by $|\cdot|$ the norm of of both $H$ and $H^{3}$, and by $\|\cdot\|_{X}$ the norm of the generic Banach space $X$. Given any $\zeta \in V^{\prime}$, let us now set

$$
\zeta_{\Omega}:=\frac{1}{|\Omega|}\langle\zeta, 1\rangle ; \quad V_{0}^{\prime}:=\left\{\zeta \in V^{\prime}: \zeta_{\Omega}=0\right\}, \quad V_{0}:=V \cap V_{0}^{\prime} .
$$

Let also $0<\alpha \leq \mu_{0}$ be assigned constants and let

$$
\mu \in \operatorname{Lip}_{\text {loc }}\left(\mathbb{R}^{2}\right), \quad \text { with } \alpha \leq \mu \leq \mu_{0} \text { a.e. in } \mathbb{R}^{2} .
$$

If $v, z: \Omega \rightarrow \mathbb{R}$ are measurable functions, then we can define the operators

$$
\begin{gathered}
B_{(v, z)}: V \rightarrow V^{\prime}, \quad\left\langle B_{(v, z)} u, y\right\rangle:=\int_{\Omega} \mu(v, z) \nabla u \cdot \nabla y d x \quad \text { for } u, y \in V, \\
B: V \rightarrow V^{\prime}, \quad\langle B u, y\rangle:=\int_{\Omega} \nabla u \cdot \nabla y d x \quad \text { for } u, y \in V .
\end{gathered}
$$


Clearly, $B, B_{(v, z)}$ map $V$ onto $V_{0}^{\prime}$ and their restrictions to $V_{0}$ turn out to be isomorphisms of $V_{0}$ onto $V_{0}^{\prime}$. Then, we can denote by $\mathcal{N}$ the inverse of $B$ and by $\mathcal{N}_{(v, z)}$ the inverse of $B_{(v, z)}$. Just by applying the definition (1.6), one can readily check that for any $u \in V$ and $\zeta \in V_{0}^{\prime}$ there holds

$$
\left\langle B_{(v, z)} u, \mathcal{N}_{(v, z)} \zeta\right\rangle=\left\langle B_{(v, z)} \mathcal{N}_{(v, z)} \zeta, u\right\rangle=\langle\zeta, u\rangle .
$$

Let now $p \in[1, \infty]$, let $v, z$ be measurable on $Q$, and let $u, \zeta$ be measurable functions of time with values in $V$ and $V_{0}^{\prime}$, respectively. Then, for a.e. $t \in(0, T)$, we can put $\left(B_{(v, z)} u\right)(t):=B_{(v(t), z(t))}(u(t))$ and $\left(\mathcal{N}_{(v, z)} \zeta\right)(t):=\mathcal{N}_{(v(t), z(t))}(\zeta(t))$. This provides a natural extension of the above operators to a time-dependent setting.

From this point on, $k$ will stand for a positive constant (possibly not always the same) depending only on $\alpha, \mu_{0}, \Omega$. A positive constant depending on one, or more, additional parameters (say, $\delta$ ) will be noted as $k_{\delta}$, instead. We have (see [4, Sec. 2] or $[1$, Sec. 2] for the proof, that is essentially based on the Poincarè-Wirtinger inequality):

Lemma 1.1. For all $\zeta \in V_{0}^{\prime}$ and for all measurable $v, z: \Omega \rightarrow \mathbb{R}$, we have that

$$
\left\|\mathcal{N}_{(v, z)} \zeta\right\|_{V} \leq k\|\zeta\|_{V^{\prime}}, \quad\left\langle\zeta, \mathcal{N}_{(v, z)} \zeta\right\rangle \geq k\|\zeta\|_{V^{\prime}}^{2}
$$

Moreover, if $v, z$ are measurable functions of $Q$ into $\mathbb{R}, p \in[1, \infty]$, then

$$
B_{(v, z)}: L^{p}(0, T ; V) \rightarrow L^{p}\left(0, T ; V_{0}^{\prime}\right) \quad \text { and } \quad \mathcal{N}_{(v, z)}: L^{p}\left(0, T ; V_{0}^{\prime}\right) \rightarrow L^{p}\left(0, T ; V_{0}\right),
$$

introduced as noted above, are well defined. In addition, the $B_{(v, z)}$ are (surjective) isomorphisms of $L^{p}\left(0, T ; V_{0}\right)$ onto $L^{p}\left(0, T ; V_{0}^{\prime}\right)$. Finally, we have that

$$
\left\|B_{(v, z)}\right\|_{\mathcal{L}\left(L^{p}(0, T ; V), L^{p}\left(0, T ; V_{0}^{\prime}\right)\right)} \leq k, \quad\left\|\mathcal{N}_{(v, z)}\right\|_{\mathcal{L}\left(L^{p}\left(0, T ; V_{0}^{\prime}\right), L^{p}\left(0, T ; V_{0}\right)\right)} \leq k,
$$

i.e., their norms do not depend on $(v, z)$.

A consequence of (1.9) and of the compact embedding $V \subset \subset H$ is that, if $\delta>0$, $\zeta \in V_{0}, \zeta=B_{(v, z)} u$ for a suitable $u \in V$, then

$$
|\zeta|^{2} \leq \delta|\nabla \zeta|^{2}+k_{\delta}\|\zeta\|_{V^{\prime}}^{2} \leq \delta|\nabla \zeta|^{2}+k_{\delta}|\nabla u|^{2} .
$$

Now, let us analyze some deeper continuity properties of the operator $\mathcal{N}_{(v, z)}$. We report the proofs, which follow the lines of [1, pp. 493-494], for the sake of completeness:

Lemma 1.2. Let $v, z \in H^{2}(\Omega), \zeta \in H \cap V_{0}^{\prime}$, and $u \in V$ be such that $\zeta:=B_{(v, z)} u$. Let also $\mu \in W^{1, \infty}\left(\mathbb{R}^{2}\right)$. Then, there exists $k>0$ such that

$$
\|u\|_{H^{2}(\Omega)} \leq k\left(|\zeta|+\|(v, z)\|_{H^{2}(\Omega)^{2}}^{2}|\nabla u|\right) .
$$

Proof. Since $\zeta \in H$, our hypotheses on $v, z, \mu$ guarantee that $u$ is in $H^{2}(\Omega)$ and satisfies

$$
-\operatorname{div}(\mu(v, z) \nabla u)=\zeta \quad \text { in } H .
$$

We deduce that $-\mu(v, z) \Delta u=(\nabla \mu(v, z)) \cdot \nabla u+\zeta$ in $H$ and consequently

$$
\int_{\Omega}|-\Delta u|^{2} \leq k|\zeta|^{2}+k \int_{\Omega}\left(|\nabla v|^{2}+|\nabla z|^{2}\right)|\nabla u|^{2} .
$$


Now, using elementary interpolation, Sobolev's embedding theorem, and Young's inequality, we obtain

$$
\begin{aligned}
\|u\|_{H^{2}(\Omega)}^{2} & \leq k\left(|u|^{2}+|-\Delta u|^{2}\right) \\
& \leq k|\zeta|^{2}+k\left(\|v\|_{H^{2}(\Omega)}^{2}+\|z\|_{H^{2}(\Omega)}^{2}\right)|\nabla u|\|u\|_{H^{2}(\Omega)} \\
& \leq k|\zeta|^{2}+k\left(\|v\|_{H^{2}(\Omega)}^{4}+\|z\|_{H^{2}(\Omega)}^{4}\right)|\nabla u|^{2}+\frac{1}{2}\|u\|_{H^{2}(\Omega)}^{2} .
\end{aligned}
$$

Lemma 1.3. Let $u, v, z, \zeta$ be functions from $Q$ into $\mathbb{R}$, with $\zeta=B_{(v, z)} u$ a.e. in $(0, T)$. Then, the following formulas hold whenever they make sense:

$$
\begin{aligned}
& \left\langle\left(B_{(v, z)} u\right)_{t}, u\right\rangle=\frac{1}{2} \frac{d}{d t}\left\langle B_{(v, z)} u, u\right\rangle+\frac{1}{2} \int_{\Omega} \mu(v, z)_{t}|\nabla u|^{2}, \\
& \left\langle B_{(v, z)} u, u_{t}\right\rangle=\frac{1}{2} \frac{d}{d t}\left\langle B_{(v, z)} u, u\right\rangle-\frac{1}{2} \int_{\Omega} \mu(v, z)_{t}|\nabla u|^{2} .
\end{aligned}
$$

Proof. Let us assume that all the functions are sufficiently regular to give sense to the integrations by parts below. Then we have

$$
\begin{aligned}
\left\langle\left(B_{(v, z)} u\right)_{t}, u\right\rangle & =\frac{d}{d t}\left\langle B_{(v, z)} u, u\right\rangle-\left\langle B_{(v, z)} u, \partial_{t} u\right\rangle \\
& =\int_{\Omega} \partial_{t}\left(\mu(v, z)|\nabla u|^{2}\right)-\frac{1}{2} \int_{\Omega} \mu(v, z) \partial_{t}|\nabla u|^{2} \\
& =\frac{1}{2} \int_{\Omega} \partial_{t}\left(\mu(v, z)|\nabla u|^{2}\right)+\frac{1}{2} \int_{\Omega} \mu(v, z)_{t}|\nabla u|^{2}
\end{aligned}
$$

so that (1.13) is proved in the regular case and in general we can conclude by a density argument. The proof of (1.14) is analogous.

\section{Main results}

Let us give the main assumptions on the data of the problem. Let $K>0$ and let

$$
\begin{aligned}
& F_{1}, F_{2}, \gamma, g \in W^{1, \infty}(\mathbb{R}), \\
& \left|F_{1}\right|,\left|F_{2}\right|,|\gamma|,|g|,\left|F_{1}^{\prime}\right|,\left|F_{2}^{\prime}\right|,\left|\gamma^{\prime}\right|,\left|g^{\prime}\right| \leq K \quad \text { a.e. in } \mathbb{R}, \\
& \varphi_{0} \in H, \quad c_{0} \in V, \\
& \beta \subset \mathbb{R} \times \mathbb{R} \quad \text { maximal monotone graph s.t. } 0 \in \beta(0), \\
& \psi\left(c_{0}\right) \in L^{1}(\Omega), \\
& c_{\Omega} \in \operatorname{int} D(\beta), \quad \text { where } \quad c_{\Omega}:=\left(c_{0}\right)_{\Omega} .
\end{aligned}
$$

Here, $\psi: \mathbb{R} \rightarrow[0,+\infty]$ is a convex and lower semicontinuous function such that $\beta=\partial \psi$ and $\psi(0)=0$ and the domain of the graph $\beta$ is required to fulfill

$$
D(\beta):=\{r \in \mathbb{R}: \quad \beta(r) \neq \emptyset\} \subset[0,1]
$$

Then, the following existence-regularity result [4, Thms. 3.1, 3.3, 3.4] holds: 
Theorem 2.1. Let assumptions (1.5), (2.1-2.7) hold. Then, there exists a quadruple $(\varphi, c, w, \xi)$ such that

$$
\begin{aligned}
& \varphi \in H^{1}\left(0, T ; V^{\prime}\right) \cap L^{2}(0, T ; V), \\
& c \in H^{1}\left(0, T ; V^{\prime}\right) \cap L^{\infty}(0, T ; V) \cap L^{2}\left(0, T ; H^{2}(\Omega)\right), \\
& w \in L^{2}(0, T ; V), \\
& \xi \in L^{2}(0, T ; H) .
\end{aligned}
$$

The quadruple $(\varphi, c, w, \xi)$ satisfies

$$
\begin{aligned}
\partial_{t} \varphi+B \varphi=F_{1}(\varphi)+c F_{2}(\varphi) & \text { in } V^{\prime}, \quad \text { a.e. in }(0, T), \\
\partial_{t} c+B_{(\varphi, c)} w=0 & \text { in } V^{\prime}, \text { a.e. in }(0, T), \\
w=B c+\xi+\gamma(c)+g(\varphi) & \text { in } V^{\prime}, \text { a.e. in }(0, T), \\
\xi \in \beta(c) & \text { a.e. in } Q, \\
\varphi(\cdot, 0)=\varphi_{0}(\cdot), \quad c(\cdot, 0)=c_{0}(\cdot) & \text { a.e. in } \Omega, \\
c(t)_{\Omega}=c_{\Omega} & \text { for all } t \in[0, T] .
\end{aligned}
$$

If, in addition,

$$
\varphi_{0} \in V
$$

then $\varphi$ satisfies

$$
\varphi \in H^{1}(0, T ; H) \cap C^{0}([0, T] ; V) \cap L^{2}\left(0, T ; H^{2}(\Omega)\right)
$$

and if

$$
\varphi_{0} \in H^{2}(\Omega), \quad \partial_{\mathbf{n}} \varphi_{0}=0 \quad \text { a.e. on } \Gamma,
$$

then $\varphi$ has the additional regularity

$$
\varphi \in W^{1, \infty}(0, T ; H) \cap H^{1}(0, T ; V) \cap L^{\infty}\left(0, T ; H^{2}(\Omega)\right) \cap L^{2}\left(0, T ; H^{3}(\Omega)\right) .
$$

Finally, just under the assumptions (1.5), (2.1-2.7), if $\mu(\varphi, c)$ is constant, then the solution $(\varphi, c, w, \xi)$ is unique.

Under more restrictive hypotheses on data, here we are able to prove additional uniqueness and regularity properties of the solution(s). We will possibly assume

$$
\begin{gathered}
\mu=\mu(\varphi) \quad \text { depends only on } \varphi, \\
\mu \in W^{1, \infty}, \quad\|\mu\|_{W^{1, \infty}} \leq K .
\end{gathered}
$$

Theorem 2.2. Let (1.5), (2.1-2.7), (2.18), and (2.22-2.23) hold for a couple of initial data $\varphi_{0,1}, c_{0,1}$ and $\varphi_{0,2}, c_{0,2}$ and assume that

$$
\left(c_{0,1}\right)_{\Omega}=\left(c_{0,2}\right)_{\Omega} .
$$

Moreover, suppose that

$$
\begin{gathered}
\partial_{t} \varphi_{1}, B \varphi_{1} \in L^{4}(0, T ; H), \\
\left\|\varphi_{1}\right\|_{L^{4}(0, T ; H)}+\left\|\partial_{t} \varphi_{1}\right\|_{L^{4}(0, T ; H)}+\left\|B \varphi_{1}\right\|_{L^{4}(0, T ; H)}+\left\|w_{2}\right\|_{L^{2}(0, T ; V)} \leq R
\end{gathered}
$$


for a given $R>0$. Then, if $\left(\varphi_{1}, c_{1}, w_{1}, \xi_{1}\right)$ and $\left(\varphi_{2}, c_{2}, w_{2}, \xi_{2}\right)$ are a couple of solutions to $(2.12-2.17)$ related to the initial data $\varphi_{0,1}, c_{0,1}$ and $\varphi_{0,2}, c_{0,2}$, respectively, we have

$$
\begin{aligned}
& \left\|\varphi_{1}-\varphi_{2}\right\|_{L^{\infty}(0, T ; V) \cap L^{2}\left(0, T ; H^{2}(\Omega)\right)}+\left\|c_{1}-c_{2}\right\|_{L^{\infty}\left(0, T ; V^{\prime}\right) \cap L^{2}(0, T ; V)} \\
& \quad \leq k_{R}\left(\left\|\varphi_{0,1}-\varphi_{0,2}\right\|_{V}+\left\|c_{0,1}-c_{0,2}\right\|_{V^{\prime}}\right) .
\end{aligned}
$$

In particular, the solution $(\varphi, c, w, \xi)$ provided by Theorem 2.1 is unique.

Remark 2.3. We note that (2.25) is surely satisfied if (2.20) holds.

Theorem 2.4. Let (1.5), (2.1-2.7), (2.20), and (2.23) hold. Let also assume that

$$
c_{0} \in H^{3}(\Omega), \quad \partial_{\mathbf{n}} c_{0}=0 \quad \text { on } \Gamma, \quad \exists \xi_{0} \in V \text { such that } \xi_{0} \in \beta\left(c_{0}\right) \text { a.e. in } \Omega \text {. }
$$

Then, there exists $T_{0} \in(0, T]$ such that the solution $(\varphi, c, w, \xi)$ given by Theorem 2.1 fulfills the additional regularity

$$
\begin{aligned}
& c \in H^{1}\left(0, T_{0} ; V\right) \cap L^{\infty}\left(0, T_{0} ; H^{2}(\Omega)\right), \\
& w \in L^{\infty}\left(0, T_{0} ; V\right) \cap L^{2}\left(0, T_{0} ; H^{2}(\Omega)\right) .
\end{aligned}
$$

Moreover, if (2.22) holds, we can choose $T_{0}=T$.

Remark 2.5. Thanks to the first of (2.29), under suitable hypotheses on $\varphi_{0}$ (e.g., $\varphi_{0} \in H^{3}(\Omega)$ and $\partial_{\mathbf{n}} \varphi_{0}=0$ on $\Gamma$ ), further parabolic regularity could be deduced also for $\varphi$ by differentiating (2.12) and multiplying the result by $\partial_{t t} \varphi, \partial_{t} B \varphi$. We do not enter into the details, since the procedure is standard.

Theorem 2.6. Let (1.5), (2.1-2.7), (2.18), and (2.23) hold for two pairs of initial data $\varphi_{0,1}, c_{0,1}$ and $\varphi_{0,2}, c_{0,2}$ also satisfying (2.24). Let also $T_{0} \in(0, T]$ and suppose $\left(\varphi_{1}, c_{1}, w_{1}, \xi_{1}\right)$ and $\left(\varphi_{2}, c_{2}, w_{2}, \xi_{2}\right)$ be a couple of solutions to $(2.12-2.17)$ related to the different initial data and fulfilling

$$
\begin{gathered}
\partial_{t} \varphi_{1}, B \varphi_{1}, \partial_{t} c_{1}, B c_{1} \in L^{4}\left(0, T_{0} ; H\right), \quad \nabla w_{2} \in L^{2}\left(0, T_{0} ; L^{3}(\Omega)\right), \\
\left\|\varphi_{1}\right\|_{L^{4}\left(0, T_{0} ; H\right)}+\left\|\partial_{t} \varphi_{1}\right\|_{L^{4}\left(0, T_{0} ; H\right)}+\left\|B \varphi_{1}\right\|_{L^{4}\left(0, T_{0} ; H\right)}+\left\|c_{1}\right\|_{L^{4}\left(0, T_{0} ; H\right)} \\
+\left\|\partial_{t} c_{1}\right\|_{L^{4}\left(0, T_{0} ; H\right)}+\left\|B c_{1}\right\|_{L^{4}\left(0, T_{0} ; H\right)}+\left\|\nabla w_{2}\right\|_{L^{2}\left(0, T_{0} ; L^{3}(\Omega)\right)} \leq R
\end{gathered}
$$

for a given $R>0$. Then, we have

$$
\begin{aligned}
& \left\|\varphi_{1}-\varphi_{2}\right\|_{L^{\infty}\left(0, T_{0} ; V\right) \cap L^{2}\left(0, T_{0} ; H^{2}(\Omega)\right)}+\left\|c_{1}-c_{2}\right\|_{L^{\infty}\left(0, T_{0} ; V^{\prime}\right) \cap L^{2}\left(0, T_{0} ; V\right)} \\
& \quad \leq k_{R}\left(\left\|\varphi_{0,1}-\varphi_{0,2}\right\|_{V}+\left\|c_{0,1}-c_{0,2}\right\|_{V^{\prime}}\right) .
\end{aligned}
$$

In particular, the solution $(\varphi, c, w, \xi)$ given by Theorem 2.1 is unique on $\left[0, T_{0}\right]$.

Remark 2.7. As before, (2.31-2.32) are not satisfied in the given regularity setting. However, if (2.20) and (2.28) are assumed, then (2.31-2.32) do hold at least up to a suitably small final time $T_{0}$. 


\section{Proofs of the Theorems}

Proof of Theorem 2.2. Set $(\varphi, c, w, \xi):=\left(\varphi_{1}, c_{1}, w_{1}, \xi_{1}\right)-\left(\varphi_{2}, c_{2}, w_{2}, \xi_{2}\right)$. Then, writing (2.12) for the two solutions and subtracting the results, we obtain

$$
\partial_{t} \varphi+B \varphi=\left(F_{1}\left(\varphi_{1}\right)-F_{1}\left(\varphi_{2}\right)\right)+c_{1}\left(F_{2}\left(\varphi_{1}\right)-F_{2}\left(\varphi_{2}\right)\right)+c F_{2}\left(\varphi_{2}\right) .
$$

Doing the same with (2.13-2.14), we have, respectively,

$$
\begin{aligned}
& \partial_{t} c+B_{\varphi_{1}} w+\left(B_{\varphi_{1}}-B_{\varphi_{2}}\right) w_{2}=0, \\
& w=B c+\xi+\left(\gamma\left(c_{1}\right)-\gamma\left(c_{2}\right)\right)+\left(g\left(\varphi_{1}\right)-g\left(\varphi_{2}\right)\right) .
\end{aligned}
$$

Then, we test (3.1) by $\varphi+B \varphi$ and integrate over $(0, t)$ for $t \leq T$. By $(2.1-2.2),(2.7)$, and Young's inequality, we easily infer

$$
\frac{1}{2}\|\varphi(t)\|_{V}^{2}+\|\nabla \varphi\|_{L^{2}\left(Q_{t}\right)}^{2}+\frac{1}{2}\|B \varphi\|_{L^{2}\left(Q_{t}\right)}^{2} \leq \frac{1}{2}\left\|\varphi_{0,1}-\varphi_{0,2}\right\|_{V}^{2}+k\|\varphi\|_{L^{2}\left(Q_{t}\right)}^{2}+k\|c\|_{L^{2}\left(Q_{t}\right)}^{2} .
$$

Indeed, (2.7) guarantees that $\left\|c_{1}\right\|_{L^{\infty}(Q)} \leq 1$.

Now, we test (3.3) by $c$ (that is in $V_{0}$ by (2.24) and (2.17)) and (3.2) by $\mathcal{N}_{\varphi_{1}} c=$ : $u$, subtract the results, and integrate over $(0, t)$. Noting that two terms cancel and using Young's inequality and the monotonicity of $\beta$, we get

$$
\begin{aligned}
& \int_{0}^{t}\left\langle\partial_{t}\left(B_{\varphi_{1}} u\right), u\right\rangle+\int_{0}^{t} \int_{\Omega}\left(\mu\left(\varphi_{1}\right)-\mu\left(\varphi_{2}\right)\right) \nabla w_{2} \cdot \nabla u+\|\nabla c\|_{L^{2}\left(Q_{t}\right)}^{2} \\
& \quad \leq k\|\varphi\|_{L^{2}\left(Q_{t}\right)}^{2}+k\|c\|_{L^{2}\left(Q_{t}\right)}^{2} .
\end{aligned}
$$

Let us deal with the first two terms $I_{1}, I_{2}$ on the left hand side. As for the first one, by $(1.13),(1.9)$, and $(2.23)$, we have

$$
\begin{aligned}
I_{1} & =\frac{1}{2} \int_{\Omega} \mu\left(\varphi_{1}(t)\right)|\nabla u(t)|^{2}-\frac{1}{2} \int_{\Omega} \mu\left(\varphi_{0,1}\right)|\nabla u(0)|^{2}+\frac{1}{2} \int_{0}^{t} \int_{\Omega} \mu^{\prime}\left(\varphi_{1}\right) \partial_{t} \varphi_{1}|\nabla u|^{2} \\
& \geq \frac{\alpha}{2}|\nabla u(t)|^{2}-\frac{\mu_{0}}{2}\left\|c_{0,1}-c_{0,2}\right\|_{V^{\prime}}^{2}-\frac{K}{2} \int_{0}^{t} \int_{\Omega}\left|\partial_{t} \varphi_{1}\right||\nabla u|^{2} .
\end{aligned}
$$

Then, using (1.12) and a Gagliardo-Nirenberg inequality [5, p. 125], we infer

$$
\begin{aligned}
& \int_{0}^{t} \int_{\Omega}\left|\partial_{t} \varphi_{1}\right||\nabla u|^{2} \leq k \int_{0}^{t}\left|\partial_{t} \varphi_{1}\right||| \nabla u \|_{L^{4}(\Omega)}^{2} \leq k \int_{0}^{t}\left|\partial_{t} \varphi_{1}\right|\left(\|u\|_{H^{2}(\Omega)}^{3 / 2}|\nabla u|^{1 / 2}+|\nabla u|^{2}\right) \\
& \leq k \int_{0}^{t}\left|\partial_{t} \varphi_{1}\right||\nabla u|^{2}+k \int_{0}^{t}\left|\partial_{t} \varphi_{1}\right||\nabla u|^{1 / 2}\left(|c|^{3 / 2}+\left\|\varphi_{1}\right\|_{H^{2}(\Omega)}^{3}|\nabla u|^{3 / 2}\right) \\
& \leq k \int_{0}^{t}\left|\partial_{t} \varphi_{1}\right||\nabla u|^{2}+k\|c\|_{L^{2}\left(Q_{t}\right)}^{2}+k \int_{0}^{t}\left(\left|\partial_{t} \varphi_{1}\right|^{4}+\left|B \varphi_{1}\right|^{4}+\left|\varphi_{1}\right|^{4}\right)|\nabla u|^{2}
\end{aligned}
$$

The second term in (3.5) thanks to the continuous embedding $H^{2}(\Omega) \subset L^{\infty}(\Omega)$ gives

$$
\left|I_{2}\right| \leq k \int_{0}^{t}\|\varphi\|_{L^{\infty}(\Omega)}\left|\nabla w_{2}\right||\nabla u| \leq \frac{1}{4} \int_{0}^{t}\left(|\varphi|^{2}+|B \varphi|^{2}\right)+k \int_{0}^{t}\left|\nabla w_{2}\right|^{2}|\nabla u|^{2} .
$$


Now, take the sum of (3.4) and (3.5) and note that (1.11) can be applied with $c$ in place of $\zeta$, so that the $L^{2}$ norms of $c$ on the right hand sides of (3.4) and (3.5) are controlled by taking an opportune $\delta$. Taking advantage of (3.6-3.8) and recalling assumptions (2.25-2.26), we conclude by applying the Gronwall lemma in the form of, e.g., [3, Lemme A.4, p. 156], to the function $t \mapsto\|\varphi(t)\|_{V}^{2}+|\nabla u(t)|^{2}$.

Proof of Theorem 2.4. We derive new a priori bounds for the solutions of $(2.12-$ 2.16). We point out that, at this level, some passages are formal. Anyway, they could be made rigorous by performing the estimates at an approximating level (cf., e.g., the Faedo-Galerkin argument of [4, Sec. 3]). Then, differentiate (2.14) in time. This gives

$$
\partial_{t} w=\partial_{t} B c+\partial_{t} \xi+\gamma^{\prime}(c) \partial_{t} c+g^{\prime}(\varphi) \partial_{t} \varphi
$$

Now, test (2.13) by $\partial_{t} w$ and (3.9) by $\partial_{t} c$ and integrate over $(0, t), t \leq T$. Noting that two terms cancel and using (1.14), (2.1-2.2), (2.23), and the monotonicity of $\beta$, we get

$$
\begin{aligned}
& \left\|\partial_{t} \nabla c\right\|_{L^{2}\left(Q_{t}\right)}^{2}+\frac{\alpha}{2}|\nabla w(t)|^{2} \leq \frac{\mu_{0}}{2}|\nabla w(0)|^{2} \\
& \quad+k \int_{0}^{t} \int_{\Omega}\left(\left|\partial_{t} \varphi\right|+\left|\partial_{t} c\right|\right)|\nabla w|^{2}+k\left\|\partial_{t} \varphi\right\|_{L^{2}\left(Q_{t}\right)}^{2}+k\left\|\partial_{t} c\right\|_{L^{2}\left(Q_{t}\right)}^{2}
\end{aligned}
$$

Of course, the main trouble is given by the integral term $J$ on the right hand side. Using Sobolev's embeddings, (1.12), and the Gagliardo-Nirenberg inequalities, we infer

$$
\begin{aligned}
J & \leq k \int_{0}^{t}\left(\left\|\partial_{t} \varphi\right\|_{V}+\left\|\partial_{t} c\right\|_{V}\right)\|\nabla w\|_{L^{12 / 5}(\Omega)}^{2} \\
& \leq k \int_{0}^{t}\left(\left\|\partial_{t} \varphi\right\|_{V}+\left\|\partial_{t} c\right\|_{V}\right)\left(|\nabla w|^{3 / 2}\|w\|_{H^{2}(\Omega)}^{1 / 2}+|\nabla w|^{2}\right) \\
& \leq k \int_{0}^{t}\left(\left\|\partial_{t} \varphi\right\|_{V}+\left\|\partial_{t} c\right\|_{V}\right) \cdot \\
& \quad\left(|\nabla w|^{3 / 2}\left|\partial_{t} c\right|^{1 / 2}+|\nabla w|^{2}\left(1+\|\varphi\|_{H^{2}(\Omega)}+\|c\|_{H^{2}(\Omega)}\right)\right)=: \sum_{j=1}^{6} J_{j}
\end{aligned}
$$

where the integrals $J_{j}$ are defined this way:

$$
\begin{array}{cc}
J_{1}:=k \int_{0}^{t}\left\|\partial_{t} \varphi\right\|_{V}|\nabla w|^{3 / 2}\left|\partial_{t} c\right|^{1 / 2}, & J_{2}:=k \int_{0}^{t}\left\|\partial_{t} c\right\|_{V}|\nabla w|^{3 / 2}\left|\partial_{t} c\right|^{1 / 2}, \\
J_{3}:=k \int_{0}^{t}\left\|\partial_{t} \varphi\right\|_{V}|\nabla w|^{2}\left(1+\|\varphi\|_{H^{2}(\Omega)}\right), & J_{4}:=k \int_{0}^{t}\left\|\partial_{t} \varphi\right\|_{V}|\nabla w|^{2}\|c\|_{H^{2}(\Omega)}, \\
J_{5}:=k \int_{0}^{t}\left\|\partial_{t} c\right\|_{V}|\nabla w|^{2}\left(1+\|\varphi\|_{H^{2}(\Omega)}\right), & J_{6}:=k \int_{0}^{t}\left\|\partial_{t} c\right\|_{V}|\nabla w|^{2}\|c\|_{H^{2}(\Omega)} .
\end{array}
$$

Now, we estimate the $J_{j}$. The term $J_{3}$ does not give troubles. Instead, $J_{1}, J_{2}, J_{4}, J_{5}$, 
owing to the third of (2.21), are bounded as follows:

$$
\begin{aligned}
& J_{1} \leq k \int_{0}^{t}|\nabla w|^{2}\left\|\partial_{t} \varphi\right\|_{V}^{4 / 3}+k \int_{0}^{t}\left|\partial_{t} c\right|^{2}, \\
& J_{2} \leq \delta \int_{0}^{t}\left\|\partial_{t} c\right\|_{V}^{2}+k_{\delta} \int_{0}^{t}|\nabla w|^{6} \\
& J_{4} \leq k \int_{0}^{t}|\nabla w|^{2}\|c\|_{H^{2}(\Omega)}^{4}+k \int_{0}^{t}|\nabla w|^{2}\left\|\partial_{t} \varphi\right\|_{V}^{4 / 3}, \\
& J_{5} \leq \delta \int_{0}^{t}\left\|\partial_{t} c\right\|_{V}^{2}+k_{\delta} \int_{0}^{t}|\nabla w|^{4} .
\end{aligned}
$$

The estimation of the latter term $J_{6}$, instead, is more complicated and we proceed similarly as in $[1$, p. 497]. More precisely, we collect in the next computation also the first term resulting from $J_{4}$ on the right hand side of (3.14). By elliptic regularity,

$$
\begin{aligned}
J_{6}+ & k \int_{0}^{t}|\nabla w|^{2}\|c\|_{H^{2}(\Omega)}^{4} \\
& \leq \delta \int_{0}^{t}\left\|\partial_{t} c\right\|_{V}^{2}+k_{\delta} \int_{0}^{t}\left(|\nabla w|^{4}\left(|c|^{2}+|B c|^{2}\right)+|\nabla w|^{2}\left(|c|^{4}+|B c|^{4}\right)\right)
\end{aligned}
$$

Then, we multiply (2.14) by $B c$. Owing to the monotonicity of $\beta$, to (2.1-2.2), and to the second of (2.9), we easily infer, a.e. in $(0, T)$,

$$
|B c|^{2} \leq k\left(1+|\nabla w|^{2}+|\nabla c|^{2}\right) \leq k\left(1+|\nabla w|^{2}\right) .
$$

Then,

$$
J_{6}+k \int_{0}^{t}|\nabla w|^{2}\|c\|_{H^{2}(\Omega)}^{4} \leq \delta \int_{0}^{t}\left\|\partial_{t} c\right\|_{V}^{2}+k_{\delta} \int_{0}^{t}\left(|\nabla w|^{2}+|\nabla w|^{6}\right) .
$$

Finally, we have to bound the norm of $w(0)$ in (3.10). Writing (2.14) for $t=0$ (this could be made rigorous by working on the discrete scheme) and recalling (2.28), we see that

$$
|\nabla w(0)|^{2} \leq k\left(1+\left\|c_{0}\right\|_{H^{3}(\Omega)}^{2}+\left\|\xi_{0}\right\|_{V}^{2}+\left\|\varphi_{0}\right\|_{V}^{2}\right) \leq k .
$$

Now, let us take (3.11-3.19) into account and go back to (3.10). If (2.22) holds, the situation is simple since $J_{2}=J_{4}=J_{5}=J_{6}=0$. Actually, we can apply (1.11) with

$$
\partial_{t} c, w, \varphi \quad \text { in place of } \zeta, u, v \text { and } z,
$$

respectively. Thus, choosing $\delta$ suitably small and recalling (2.21), we see that Gronwall's Lemma applies to the function $t \rightarrow|\nabla w(t)|^{2}$. This yields $\nabla w \in L^{\infty}(0, T ; H)$ and also the first of (2.29) for $T_{0}=T$. The second of (2.29) is a consequence of (3.17). Now, the first of (2.30) would easily follow once we show that $w \in L^{\infty}\left(0, T_{0} ; H\right)$. Due to the Neumann conditions, this is not obvious and has to be proved by suitably adapting the argument of, e.g., [2, Subsec. 5.3]. At this point, also the second of (2.30) is proved since, looking back at (2.13), we can exploit (1.12) with the choices (3.20) and take advantage of the third of (2.21) and of the second of (2.29). 
Instead, if (2.22) does not hold, we deduce from (3.10-3.19) that

$$
\left\|\partial_{t} \nabla c\right\|_{L^{2}\left(Q_{t}\right)}^{2}+|\nabla w(t)|^{2} \leq k+k \int_{0}^{t}\left(f|\nabla w|^{2}+|\nabla w|^{6}\right),
$$

where $f:=\left(1+\left\|\partial_{t} \varphi\right\|_{V}^{4 / 3}\right) \in L^{p}(0, T)$ for some $p>1$. Then, using a generalized Gronwall's lemma (e.g., a slightly modified version of [6, Thm. 7.1] works for our case), we obtain the same relations as before, holding now up to a final time $T_{0} \in(0, T]$.

Proof of Theorem 2.6. It is very similar to the proof of Theorem 2.2. Then, we just give the highlights. Proceeding as in that proof (but choosing now $t \in\left(0, T_{0}\right]$ ), we see that the last term in (3.6) now depends also on $\partial_{t} c_{1}$. However, it can still be estimated as in (3.7) by taking the contribution of $\partial_{t} c_{1}$ into account. Thus, the last term in (3.7) will now depend on all the norms in (2.32) but the latter.

The bound (3.8) needs to be modified more carefully. Actually, we now have

$$
\begin{aligned}
\left|I_{2}\right| & =\left|\int_{0}^{t} \int_{\Omega}\left(\mu\left(\varphi_{1}, c_{1}\right)-\mu\left(\varphi_{2}, c_{2}\right)\right) \nabla w_{2} \cdot \nabla u\right| \\
& \leq k \int_{0}^{t}\|\varphi\|_{L^{\infty}(\Omega)}\left|\nabla w_{2}\right||\nabla u|+k \int_{0}^{t}\|c\|_{L^{6}(\Omega)}\left\|\nabla w_{2}\right\|_{L^{3}(\Omega)}|\nabla u| .
\end{aligned}
$$

Thus, the first term can be bounded as before, while the second one is

$$
\leq \delta\|c\|_{L^{2}(0, T ; V)}^{2}+k_{\delta} \int_{0}^{t}\left\|\nabla w_{2}\right\|_{L^{3}(\Omega)}^{2}|\nabla u|^{2}
$$

so that, on account of the last of (2.32), a further application of the Gronwall lemma [3, Lemme A.4, p. 156] permits us to conclude.

\section{References}

[1] J. W. Barrett and J. F. Blowey, Finite element approximation of the Cahn-Hilliard equation with concentration dependent mobility, Math. Comp., 68 (1999), 487517.

[2] E. Bonetti, W. Dreyer, and G. Schimperna, Global solution to a viscous CahnHilliard equation for tin-lead alloys with mechanical stresses, submitted.

[3] H. Brezis, "Opérateurs Maximaux Monotones et Sémi-groupes de Contractions dans les Espaces de Hilbert", North-Holland Math. Studies, 5, North-Holland, Amsterdam, 1973.

[4] D. Kessler, J.-F. Scheid, G. Schimperna, and U. Stefanelli, Study of a system for the isothermal separation of components in a binary alloy with change of phase, paper in preparation.

[5] L. Nirenberg, On elliptic partial differential equations, Ann. Scuola Norm. Sup. Pisa (3), 13 (1959), 115-162.

[6] M. Sassetti and A. Tarsia, On a nonlinear vibrating-string equation, Ann. Mat. Pura Appl. (4), 161 (1992), 1-42. 